\section{Re: Patient Beliefs Have a Greater Impact Than Barriers on Medication Adherence in a Community Health Center}

To the Editor: The article by Gagon et $\mathrm{al}^{1}$ regarding patient beliefs and barriers in medication adherence provided an introductory look into the critical need for health care providers to assess and discuss negative beliefs regarding medications when implementing a medication regimen. The article provided several key findings regarding the effect of patient beliefs and barriers on medication adherence; however, the study could have been further enhanced with some minor improvements. The majority of the participants in the study were of African-American or Hispanic background, which could indicate that cultural background has an effect on patient beliefs leading to variations in medication adherence. The discussion failed to mention any cultural implications of this study. Given that the US Census Bureau ${ }^{2}$ estimates that by the year 2060, 20\% of the US population will be foreign born and by the year 2044, 50\% of all Americans will belong to a minority group, it is important for the authors to consider the impact of cultural beliefs on individual health and medication adherence. The authors did not give recommendations for future studies which could be further enhanced by examining specific cultures such as Hispanic/Latinos, Asian-Americans, Native-Americans, or Middle-Eastern heritage, as well as specific beliefs regarding medications pertaining to these cultures.

Furthermore, the selection of participants to be included in the study may have resulted in sampling bias. Although the study examined patients reporting to the health center for regularly scheduled appointments, this subgroup of participants may inherently be more compliant than the general population as they are not only scheduling regular appointments, but also regularly attending these appointments and thus not representative of the general population. The study's primary outcome was to evaluate patient adherence to medication and specifically examined prescribed medication taken regularly by using the 8-item Morisky Medication Adherence Scale, but the authors failed to indicate what the scale specifies as "regular" when taking medications and whether missing 1 dose of medication per week versus missing 1 dose of a medication monthly is still considered taking prescribed medications regularly.
Next, the study also made note that younger individuals (18 to 25 years) had lower adherence rates compared with the other age categories. However, the authors never gave any explanation for these findings. For example, it is possible that these findings may be due to personality characteristics attributable to that age range, or because individuals that age are less likely to be taking multiple medications, all which are important for providers to consider. The study concludes that providers need to address negative beliefs held by patients to increase adherence and decrease the morbidity and mortality associated with chronic disease, yet the authors did not give any recommendations for providers for addressing negative beliefs. The discussion section of the study would have been further enhanced had they provided information for providers regarding how to address beliefs in patients and how to eliminate misunderstanding, while remaining culturally sensitive. The Agency for Health care Research and Quality by the US Department of Health and Human Services ${ }^{3}$ gives several recommendations useful to providers in addressing health beliefs.

Susana Romero-Martinez College of Health Care Sciences Nova Southeastern University Fort Myers, FL sr2002@mynsu.nova.edu

\section{References}

1. Gagnon MD, Waltermaurer E, Martin A, Friedenson C, Gayle E, Hauser DL. Patient beliefs have a greater impact than barriers on medication adherence in a community health center. J Am Board Fam Med 2017;30:331336.

2. Colby SL, Ortman JM. Projections of the size and composition of the U.S. population: 2014 to 2060. United States Census Bureau; 2005. Available from: https://www.census. gov/content/dam/Census/library/publications/2015/demo/ p25-1143.pdf. Accessed July 4, 2017.

3. U.S. Department of Health and Human Services Agency for Healthcare Research and Quality. Health literacy universal precautions toolkit. 2nd ed. Available from: https://www. ahrq.gov/sites/default/files/wysiwyg/professionals/qualitypatient-safety/quality-resources/tools/literacy-toolkit/ healthlittoolkit2_tool10.pdf. Accessed July 4, 2017.

doi: 10.3122/jabfm.2017.06.170296 